Article

\title{
Novel SU-8/Ionic Liquid Composite for Tribological Coatings and MEMS
}

\author{
Leili Batooli ${ }^{1, *}$, Sandra Guadalupe Maldonado ${ }^{1}$, Moshe Judelewicz ${ }^{2}$ and Stefano Mischler ${ }^{1}$ \\ 1 Tribology and Interface Chemistry Group (SCI-STI-SM), Ecole Polytechnique Fédérale de Lausanne \\ (EPFL), CH-1015 Lausanne, Switzerland; E-Mails: sandra.guadalupemaldonado@epfl.ch (S.G.); \\ stefano.mischler@epfl.ch (S.M.) \\ 2 Gersteltec Sàrl, Avenue Général-Guisan 26, CH-1009 Pully, Switzerland; E-Mail: moshe@gersteltec.ch \\ * Author to whom correspondence should be addressed; E-Mail: leili.batooli@gmail.com; \\ Tel.: +41-78-9133191.
}

Academic Editor: Jeong-Bong Lee

Received: 25 March 2015 / Accepted: 13 May 2015 / Published: 19 May 2015

\begin{abstract}
Tribology of SU-8 polymer is increasingly relevant due to bursting use of this material in a variety of applications. This study is directed towards introduction and investigation of a novel self-lubricating composite of an ionic liquid (IL) in SU-8. The new material can be utilized for fabrication of lubricating polymer coating with tunable surface properties or SU8-made elements for microelectromechanical systems (MEMS) with enhanced tribological performance. It is shown that addition of IL drastically alters water affinity of the composite while UV patternability remains unmodified. A lower coefficient of friction and wear has been obtained for two investigated compositions with 4 and 10 wt $\%$ ionic liquid.
\end{abstract}

Keywords: SU-8; tribology; ionic liquid; composite; MEMS

\section{Introduction}

SU-8, an epoxy-type ultraviolet (UV) sensitive negative photoresist has attracted increasing attention since its introduction by IBM in 1989. This photoresist, initially used for photolithography, soon was used as resist in X-ray lithography [1] e-beam lithography [2] and as mold material for soft lithography of thermosetting elastomers such as poly(dimethyl siloxane) (PDMS) [3]. Advantageous properties of this material including biocompatibility [4], relative ease of fabrication methods, high chemical [5], 
mechanical [5] and thermal stability after processing [4,5] have found main applications in microelectromechanical systems (MEMS) and coatings and yet, applications are rapidly emerging today.

SU-8 can also be used for fabrication of molds for polymers. Examples include replica molding of poly(methyl methacrylate) (PMMA) and also casting molds for PDMS with many applications in microfluidics and chemical sensors [6,7]. However, SU-8 molds have the downside of early signs of delamination because of the stiction between the mold and substrate [8].

Tribological properties, such as adhesion and wear, have a major effect on determining reliability of MEMS [9]. Yet, despite vast application of SU-8 material in this domain only few investigations have been performed to address tribological issues of SU-8 resin. Based on the investigations of SU-8 counteracting tribological properties, three main approaches can be distinguished: surface texturing, external lubrication and bulk modification. The first category operates on the general basis that reduced area of contact can potentially lower the friction. Osborn et al. have used connected and isolated microstructures of SU-8, with and without diamond-like carbon (DLC) coating (deposited on top of the SU-8) that acted as an outer, protective layer to improve the wear resistance. They obtained decreased coefficient of friction (COF) in rubbing tests against chrome steel ball by texturing isolated microstructured on SU-8 surface [10]. However, texturing methods generally include multi-step complicated microfabrication process and require advanced cleanroom technology.

The second approach, external lubrication, implies surface functionalization of SU-8 by low shear strength thin films acting as lubricants. For example, Singh et al., deposited lubricating perfluoropolyether (PFPE) to SU-8 surface through either physical or chemical surface modification and obtained lower coefficient of friction and wear [11]. However, integrating the lubricant only to surface and through a pre-application process comes with a hitch: once the material starts to wear, lubricant will be removed from the surface. Moreover, since no replacement source is anticipated, lubrication can be demolished.

In the third method, SU-8 is blended with other compounds to fabricate a composite with enhanced tribological properties. Mixing of SU-8 with other compounds before processing is in general a very convenient way of tuning bulk and/or surface properties. Several properties of SU-8 such as electrical [12], piezoelectric [13] and tribological [14] have been manipulated by compositing SU-8 with additives. However, to our knowledge, no investigations have so far been conducted on improving tribological properties of SU-8 through compositing with lubricating materials.

Ionic liquids are potentially interesting substances for lubricating technology. The term Ionic Liquid (IL) refers to a class of salts that are liquid in their pure state at near ambient temperature. These liquids are solely made of ions and in most cases have an organic cation and an inorganic, poly-atomic anion. Properties that make Ionic Liquids attractive materials in the field of chemistry and tribology include but are not limited to: marginal vapor pressure, good thermal stability (considerable gap between the melting point and the temperature of decomposition), characteristic electrical conductivity of an ionic conductor, low flammability, good dissolution with many organic and inorganic compounds and a wide variety of possible anions and cations combinations [15]. Based on the extensive research invested on this material class, ILs have found emerging applications in sensors, solar cells, solid-state photocells, batteries, as thermal fluids, lubricants, solvents, reaction media, hydraulic fluids to name only a few [16]. 
In summary, a material that presents desirable surface properties together with compatibility with MEMS fabrication processes can be very beneficial for many applications. Accordingly, the goal of the present work is to bring together advantageous properties of SU-8 and ionic liquids to overcome tribological shortcomings of the epoxy material by producing a composite material composed of an ionic liquid dispersed in a SU-8 matrix. In order to evaluate the potential of this composite for application in tribological coatings and MEMS, characteristics of the material including compatibility with photolithography process, wettability, and tribological properties, have been studied. Likely applications include but are not limited to self-lubricating coatings, structural MEMS material for moving parts and molds. In this study, two compositions of 4 and $10 \mathrm{wt} \%$ IL have been investigated.

\section{Experimental Section}

\subsection{Materials and Sample Preparation}

SU-8 epoxy grade GM1060 was supplied by Gersteltec Sarl, Pully, Switzerland. The ionic liquid used in this study was 1-Methyl-3-octylimidazolium hexafluorophosphate with purity $\geq 95.0 \%$ acquired from Sigma Aldrich. Schematics of both SU-8 and IL molecule used in this study are shown in Figure 1. Glass substrate used for spin coating was obtained by cutting microscope slides with thickness of $1 \mathrm{~mm}$ into rectangles of $3 \mathrm{~cm} \times 3 \mathrm{~cm}$. The pieces were then thoroughly cleaned with soap and distilled water, rinsed with isopropanol and dried with nitrogen jet.

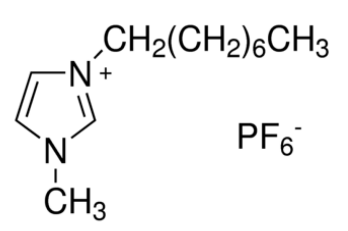

(a)

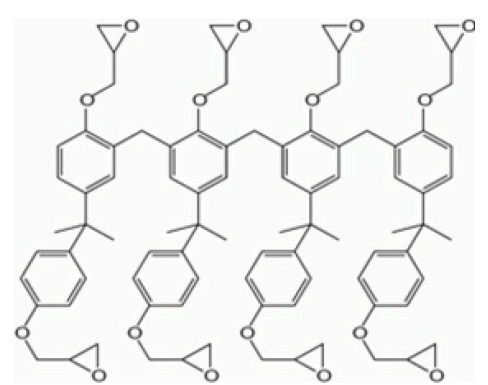

(b)

Figure 1. Schematics of (a) 1-Methyl-3-octylimidazolium hexafluorophosphate and (b) SU-8 molecules.

Composite fabrication method in this case is of crucial importance. Ideal lubrication is anticipated to occur as a result of homogeneous dispersion in forms of micro scale droplets in polymer matrix [9]. Full dissolution of IL molecules in the epoxy matrix would hinder formation of a liquid IL lubricating film while large IL aggregations would prevent homogenous IL distribution over the entire rubbing surface and thus continuous lubrication.

A thickness of 50 micron neat SU-8 or SU-8/IL composite was spin coated at $565 \mathrm{rpm}$ on the glass substrate followed by solvent evaporation at $120{ }^{\circ} \mathrm{C}$ and UV irradiated by a $7 \mathrm{~mW} / \mathrm{cm}^{2}$ Lamp. Final step of post exposure bake was performed at $95^{\circ} \mathrm{C}$ for $45 \mathrm{~min}$ to increase the crosslinking degree.

In this study, four mixing methods namely ultrasonification, rolling, mechanical mixing and magnetic stirring were evaluated. However, only mechanical mixing and magnetic stirring provided reproducible results. Following paragraphs will only be representing characterization of composites prepared via mechanical mixing. 


\subsection{X-Ray Photoelectron Spectroscopy (XPS)}

The X-Ray Photoelectron Spectroscopy (XPS) measurements were performed using a Kratos AXIS ultrahigh-resolution spectrometer (Karatos Analytical Ltd, Manchester, UK). The machine uses a monochromatic $\mathrm{Al} K_{\alpha}$ X-ray source and a take-off angle of $0^{\circ}$ with respect to the surface normal. Curve fitting was performed using the commercial software package CasaXPS (Casa Software Ltd, Teignmouth, UK).

\subsection{Wettability Characterization}

Taking into account wide applications of SU-8 in lab-on-chips and microfluidics, it is certainly relevant to investigate effect of addition of the ionic liquid on water affinity of the epoxy material. In order to measure the contact angle, the water droplet method was used. The set up consists of a microscope with a numeric camera connected to a computer and a table to translate $X Y Z$ position that was placed in front of the microscope. A volume of $2 \mu \mathrm{L}$ is deposited using a pipet. The contact angle was then manually measured on the captured photo. In each case measurements were performed twice.

\subsection{UV Pattern-Ability}

For applications in MEMS, the affordable resolution is key information in defining limitations in size and shape of structures and accordingly must be considered in design. Consequently, effect of IL on UV pattern-ability of the composite has been examined. Samples followed the same preparation steps as previously described and were exposed using a chromium mask with basic rectangular patterns. Following post-exposure bake, samples were developed in propylene glycol methyl ether acetate (PGMEA) during approximately $3 \mathrm{~min}$.

\subsection{Sliding Friction Apparatus and Test Conditions}

Tribological tests were performed using a sliding friction apparatus (SFA) previously described in [17]. Samples were rubbed against a carbon steel ball with diameter of $6 \mathrm{~mm}$ at room temperature and under relative humidity of $50 \%-60 \%$. A normal force of $2 \mathrm{~N}$ was applied over duration of $15 \mathrm{~min}$ or $60 \mathrm{~s}$ with a frequency of $2 \mathrm{~Hz}$ and a sliding velocity of $20 \mathrm{~mm} / \mathrm{s}$. A computer equipped with LabView program (home-made) was in charge of constant monitoring of normal force, frictional force and pin displacement. Coefficient of friction was calculated by dividing frictional force by normal force measured in the middle of wear track. Tests were repeated two times to check for reproducibility of results.

According to Hertz contact stress theory for elastic contact between a sphere and a plane, stress at contact depends on normal load, contact area, Young's modulus and Poisson's ratio of the two materials in contact. Properties of SU-8, including mechanical properties, are highly dependent on process parameters. For Young's Modulus, values ranging between 2.4 and 5.5 GPa have been measured depending on both process and test conditions [18]. The Poisson ratio at room temperature has also been reported to vary between 0.3 and 0.4 [19]. For the sake of following calculations, Young's modulus and Poisson's ratio have been considered to be equal to $4 \mathrm{GPa}$ and 0.3 , respectively. Steel ball had a diameter of $6 \mathrm{~mm}$ and applied normal force was equal to $2 \mathrm{~N}$. For the above-mentioned conditions, the maximum contact pressure is calculated to be equal to $92.8 \mathrm{MPa}$. Such severe pressure 
is aimed at performing accelerated tests. Note that the contact pressure was calculated considering the bulk properties of SU-8. In case of a SU-8 film deposited on a much more rigid glass substrate, the contact pressure is expected to be higher, thus yielding even more severe test conditions.

\subsection{Wear Calculation}

Wear track topography was studied using SmartWLI-basic white-light interferometer. 3D profiles were then analyzed using MountainsMap analysis software. For wear rate calculation purposes, 2D linear profiles of wear track cross section were obtained at three points over the length of the wear track. Averaged cross-sectional area obtained from these three measurements was then multiplied by the wear track length to determine the wear volume.

\section{Results}

\subsection{X-Ray Photoelectron Spectroscopy (XPS) Analysis}

Film surface has been studied using X-ray photoelectron spectroscopy. Figure 2a corresponds to pristine SU-8 and exhibits characteristic peaks of Oxygen $(\mathrm{O})$ and Carbon $(\mathrm{C})$. Whereas the spectrum performed on the composite with $4 \mathrm{wt} \%$ ionic liquid that is shown in Figure $2 \mathrm{~b}$, shows the peaks corresponding to fluorine and nitrogen, i.e., the main components of the ionic liquid aside from carbon and oxygen.
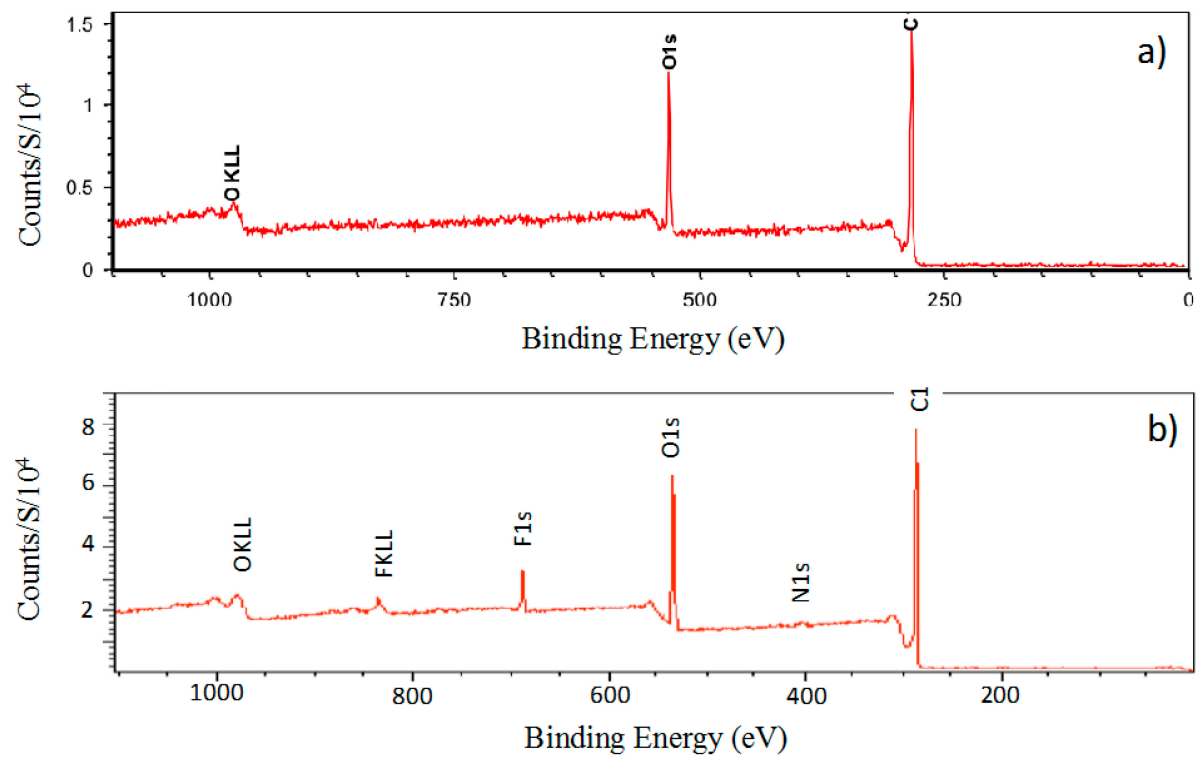

Figure 2. X-Ray Photoelectron Spectroscopy (XPS) analysis of (a) SU-8 film surface and (b) composite surface with $4 \mathrm{wt} \%$ ionic liquid (IL).

\subsection{Wettability Characterization}

According to Wenzel's model for homogeneous wetting regime [20], wetting behavior depends on surface roughness. The contact angle also depends on the manner of surface preparation and surface cleanliness. Accordingly, different authors, depending on sample preparation method, have measured different water contact angles. However, it is globally agreed that SU-8 as an organic material, is 
weakly hydrophobic with contact angle of cured resin $>70^{\circ}$ [21]. Contact angles were measured for water on pristine SU-8 and two compositions containing ionic liquid. The obtained results are presented on Figure 3. In agreement with the literature, pristine SU-8 presents a high contact angle of $79^{\circ}$ while both composites present a more hydrophilic behavior with contact angles of $57^{\circ}$ and $53^{\circ}$ for $4 \%$ and $10 \%$ IL content, respectively. The above-mentioned values are the average of two measurements that are shown in Figure 3.

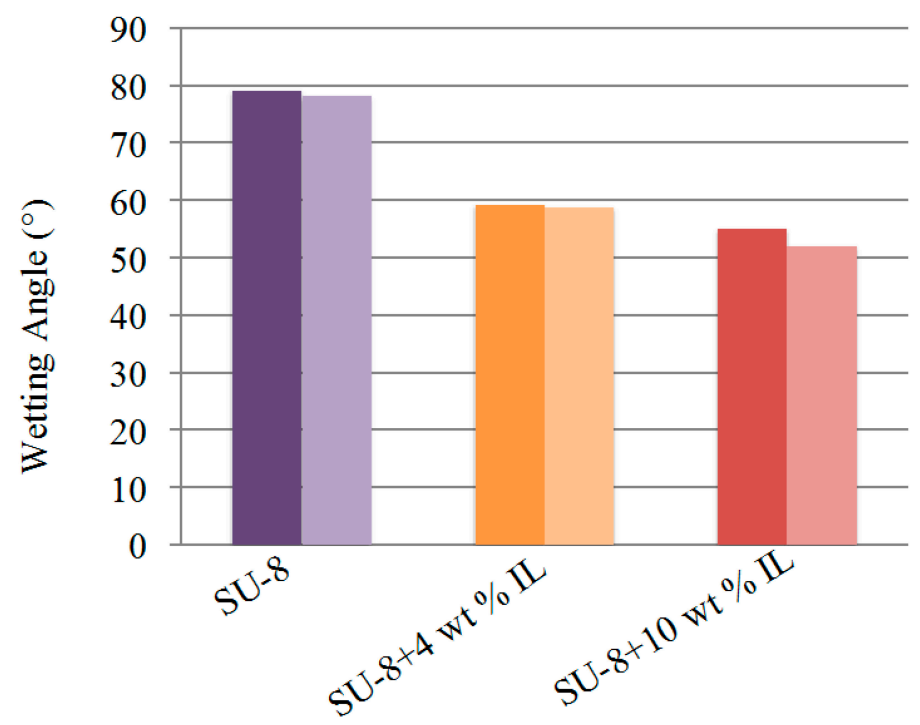

Figure 3. Wetting angles of water droplets on different surfaces. Each bar represents an independent measurement.

\subsection{UV Pattern-Ability}

Figure 4 presents optical micrographs of microstructures patterned via UV exposure on both pristine SU-8 and SU-8/IL composite. Rectangular patterns and sharp edges are attained and results are comparable for both materials.

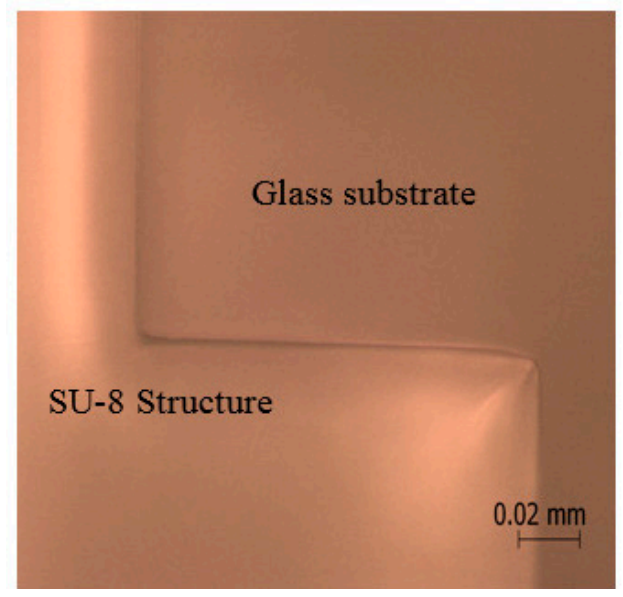

(a)

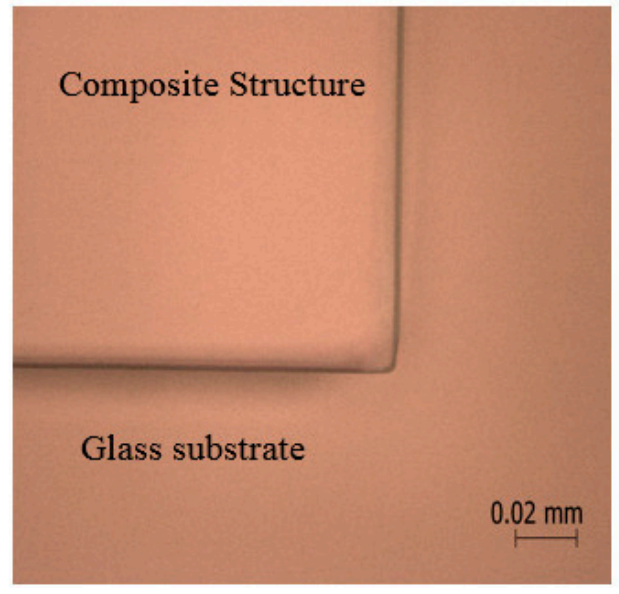

(b)

Figure 4. Optical micrograph of UV-patterned microstructures on (a) pristine SU-8 and (b) composite with $4 \mathrm{wt} \%$ ionic liquid. 


\subsection{Tribological Analysis}

3D topographical profile of the wear track surface after 1800 sliding cycles (15 min) are represented in Figure 5. It can be seen that ridges formed by accumulated wear debris surround the wear groove. Wear volume has also been calculated for the above mentioned wear tracks by measuring cross sectional area and results are presented in Figure 6. A noticeable decrease in wear volume as a result of IL addition is evident. The effect is more pronounced for higher IL content.

From Figure 5 it is evident that during the wear process, particles detach from the polymer surface. These debris particles then act as third body and dominate the wear process. Accordingly and in order to eliminate the effect caused by wear debris, sliding friction tests are performed over $60 \mathrm{~s}$ (120 cycles) to see the evolution of COF in first steps.

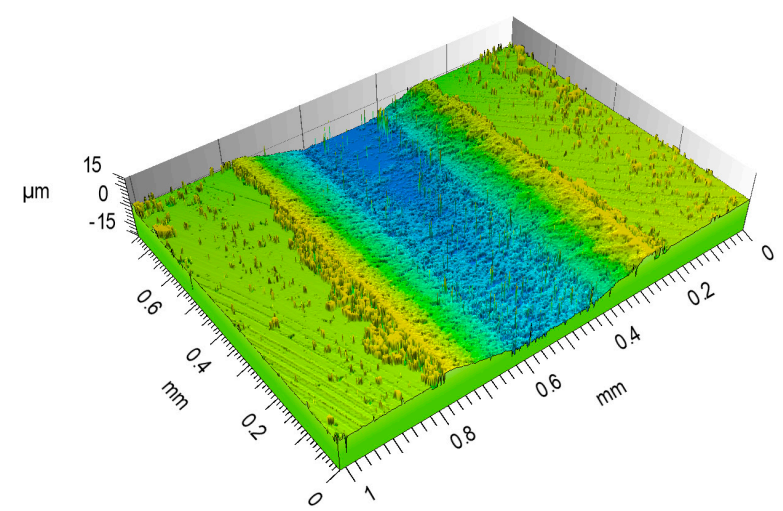

(a)
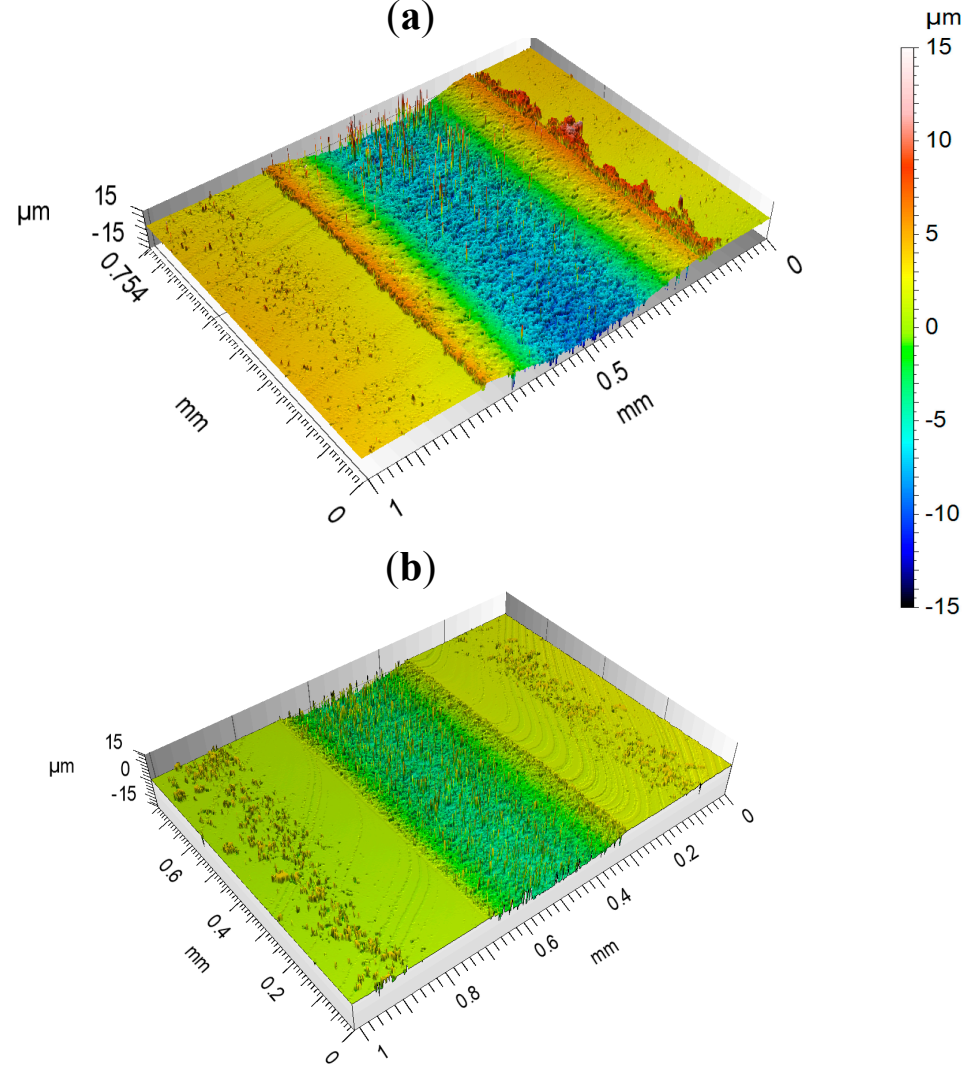

(c)

Figure 5. Optical 3D micrographs of wear tracks after 1800 cycles of (a) SU-8, (b) SU-8 + 4 wt \% IL and (c) SU-8 + $10 \mathrm{wt} \%$ IL. 


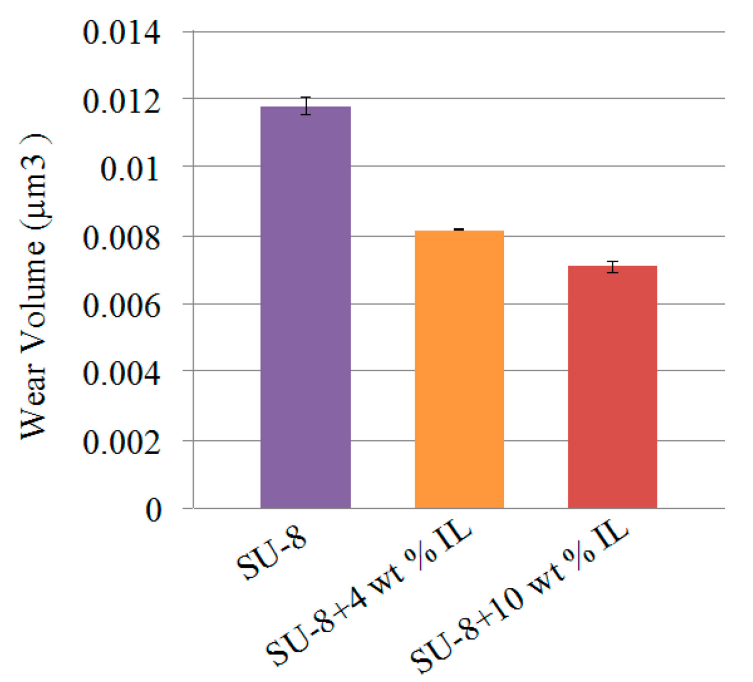

Figure 6. Wear volume after 1800 cycles for pristine SU-8, composite with $4 \%$ and $10 \%$ IL. A decreasing trend is observed as a result of addition of lubricant.

In Figure 7 evolution of coefficient of friction (COF) is plotted versus time for pristine SU-8, composite with $4 \mathrm{wt} \% \mathrm{IL}$ and $10 \mathrm{wt} \%$ IL. Until approximately $10 \mathrm{~s}$ the coefficient of friction remains at very low values possibly because of the presence of adsorbed species such as water or other surface contaminants. Pristine SU-8 shows an instant increase in COF and reaches a steady state only $20 \mathrm{~s}$ after beginning of the experiment. In contrast, evolution of COF has a gradual trend for both composites. Coefficient of friction of composite with $4 \mathrm{wt} \% \mathrm{IL}$, however, takes off even more progressively compared to the $10 \%$ and shows negligible increase in first $40 \mathrm{~s}$ of test period. After $100 \mathrm{~s}$ the coefficient of friction reached a steady state value lying between 0.5 and 0.6 similar for all tested materials. Note that hardly any wear could be observed on the IL composite specimen tested for 60 s only (only isolated scratches could be observed on the rubbed surfaces). Instead, the pristine SU-8 samples exhibited wear (material removal) already after $60 \mathrm{~s}$ of sliding. This indicates that the onset of wear is associated to the stabilization of the coefficient of friction at higher values around 0.5 .

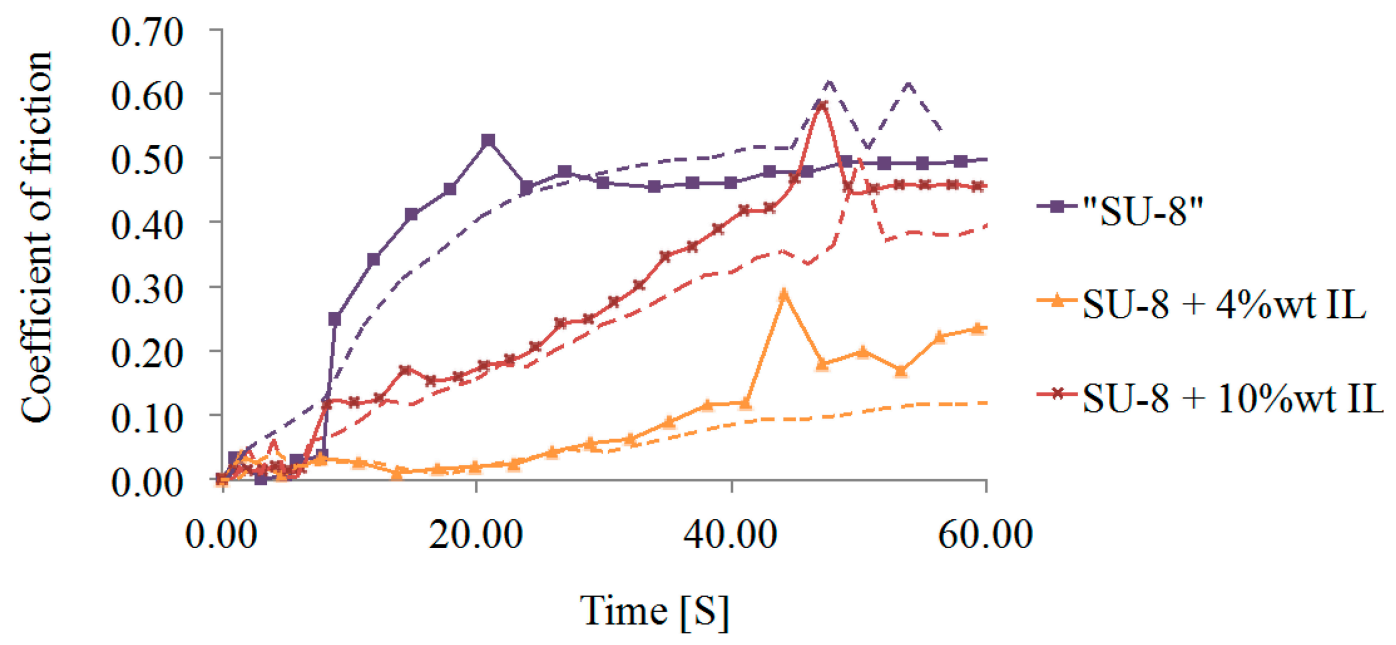

Figure 7. Evolution of coefficient of friction (COF) versus time for pristine SU-8, SU-8 with 4 wt \% IL and SU-8 with $10 \mathrm{wt} \%$ IL. For each material, test repetition is represented as dashed line of the same colour as the first test. 


\section{Discussion}

Contact angle measurement illustrates that addition of IL drastically affects water affinity of SU-8. Only by addition of 4\% IL, SU-8 surface shifts towards a more hydrophilic behavior with the contact angle dropping to approximately 60 degrees. However, the relationship is not linear and further addition of IL up to $10 \%$ induces only small decrease of 2 degrees in contact angle. A low contact angle is in particular favorable for applications in microfluidics where capillary pressure is linearly related to cosine of contact angle. For the high thickness of film (50 microns) and the soft contact imposed between the mask and the film in this experiment, obtained resolution, as determined using optical microscopy, for SU-8/IL composite is comparable to that of pristine SU-8.

Sever wear has occurred for all three tested compositions over a test period of $15 \mathrm{~min}$ and a drastic amount of debris is formed. Yet, wear is effectively reduced as a result of IL addition and increasing amount of IL from $4 \mathrm{wt} \%$ to $10 \mathrm{wt} \%$ enhances this effect. Addition of IL has effectively reduced the wear of the material.

Addition of internal lubricant in both cases of 4 and 10 percent has effectively delayed start of wear process. This effect is expressed by a gradual increase in COF vs. time for the composite in contrast with the jump for pristine SU-8. It is also observed that the composite with a lower amount of additive has more successfully reduced COF.

On the whole it is observed that increasing IL content from $4 \%$ to $10 \%$ results in more effective wear reduction. However, the lubricating effect, which is symbolized by lower coefficient of friction, is more prevailing for lower lubricant content. This contrast can be explained through the double edged sward effect of ionic liquid in the SU-8 matrix. It is assumed that the ionic liquid, along-side lubrication, affects mechanical properties of SU-8. Meaning composite with lower IL content is more mechanically robust and consequently more successful in delaying start of wear. Yet, once wear and material deterioration starts, IL that is constrained in polymer matrix is released to wear track. At this point, the higher amount of lubricant that is released to the contact area of the two rubbing surfaces dominates wear process and compensates for lower mechanical robustness.

\section{Conclusions}

A novel self-lubricating SU-8 composite, compatible with photolithography process is fabricated. Properties of this composite have been investigated to assess its potential in applications such as self-lubricating coatings, structural MEMS material for moving parts and molds material and promising results have been obtained. Two different compositions of $4 \mathrm{wt} \%$ and $10 \mathrm{wt} \%$ of IL in SU-8 are investigated and following conclusions have been drawn:

- Addition of IL shifts water affinity of SU-8 surfaces towards more hydrophilic behavior.

- $\quad$ Presence of IL does not affect the material compatibility with photolithography process.

- Wear is effectively reduced as a result of addition of IL and this effect is proportional to the amount of ionic liquid inside the composite.

- Similarly, the coefficient of friction of the composite is lower than that of SU-8 at least during an initial period. This effect is, however, more pronounced for lower percentage of IL additive. 


\section{Acknowledgments}

The study was funded through internal EPFL resources. Gersteltec Sàrl kindly provided SU-8 material and sponsored the open access publication.

\section{Author Contributions}

All authors conceived and designed the experiments and analyzed the data; Leili Batooli performed the experiments; Moshe Judelewicz supplied reagents and materials; all authors contributed to the writing of the paper.

\section{Conflicts of Interest}

The authors declare no conflict of interest.

\section{References}

1. Mekaru, H. Performance of SU-8 Membrane Suitable for Deep X-Ray Grayscale Lithography. Micromachines 2015, 6, 252-265.

2. Bilenberg, B.; Jacobsen, S.; Schmidt, M.S.; Skjolding, L.H.D.; Shi, P.; Bøggild, P.; Tegenfeldt, J.O.; Kristensen, A. High resolution $100 \mathrm{kV}$ electron beam lithography in SU-8. Microelectron. Eng. 2006, 83, 1609-1612.

3. Natarajan, S.; Chang-Yen, D.A.; Gale, B.K. Large-area, high-aspect-ratio SU-8 molds for the fabrication of PDMS microfluidic devices. J. Micromech. Microeng. 2008, 18, 045021.

4. Nemani, K.V.; Moodie, K.L.; Brennick, J.B.; Su, A.; Gimi, B. In vitro and in vivo evaluation of SU-8 biocompatibility. Mater. Sci. Eng. 2013, 33, 4453-4459.

5. Bhushan, B.; Liu, H. Nanoscale Boundary Lubrication Studies. In Nanotribology and Nanomechanics; Bhushan, P.B., Ed.; Springer: Berlin, Germany, 2005; pp. 929-962.

6. Chan-Park, M.B.; Zhang, J.; Yan, Y.; Yue, C.Y. Fabrication of large SU-8 mold with high aspect ratio microchannels by UV exposure dose reduction. Sens. Actuators B Chem. 2004, 101, 175-182.

7. Dy, A.J.; Cosmanescu, A.; Sluka, J.; Glazier, J.A.; Stupack, D.; Amarie, D. Fabricating microfluidic valve master molds in SU-8 photoresist. J. Micromech. Microeng. 2014, 24, 057001.

8. Moresco, J.; Clausen, C.H.; Svendsen, W. Improved anti-stiction coating of SU-8 molds. Sens. Actuators B Chem. 2010, 145, 698-701.

9. Saravanan, P.; Satyanarayana, N.; Sinha, S.K. Wear durability study on self-lubricating SU-8 composites with perfluoropolyther, multiply-alkylated cyclopentane and base oil as the fillers. Tribol. Int. 2013, 64, 103-115.

10. Osborn, L. Enhanced tribological properties of surfaces patterned with SU8/DLC microstructures. The Inquiry 2013, 15, 69-86.

11. Singh, R.A.; Satyanarayana, N.; Kustandi, T.S.; Sinha, S.K. Tribo-functionalizing Si and SU8 materials by surface modification for application in MEMS/NEMS actuator-based devices. J. Phys. D Appl. Phys. 2011, 44, 015301.

12. Majidian, M.; Grimaldi, C.; Pisoni, A.; Forró, L.; Magrez, A. Electrical conduction of photo-patternable SU8-graphene composites. Carbon 2014, 80, 364-372. 
13. Kandpal, M.; Sharan, C.; Poddar, P.; Prashanthi, K.; Apte, P.R.; Rao, V.R. Photopatternable nano-composite $(\mathrm{SU}-8 / \mathrm{ZnO})$ thin films for piezo-electric applications. Appl. Phys. Lett. 2012, $101,104102$.

14. Jiguet, S.; Judelewicz, M.; Mischler, S.; Hofmann, H.; Bertsch, A.; Renaud, P. SU-8 nanocomposite coatings with improved tribological performance for MEMS. Surf. Coat. Technol. 2006, 201, 2289-2295.

15. Saravanan, P.; Satyanarayana, N.; Sinha, S.K. Self-lubricating SU-8 nanocomposites for microelectromechanical systems applications. Tribol. Lett. 2013, 49, 169-178.

16. Smiglak, M.; Metlen, A.; Rogers, R.D. The Second Evolution of Ionic Liquids: From Solvents and Separations to Advanced Materials Energetic Examples from the Ionic Liquid Cookbook. Acc. Chem. Res. 2007, 40, 1182-1192.

17. Guadalupe, S.; Mischler, S.; Walter-John, C.; Carole, F. Effect of Dwell Time and Electrode Potential on the Tribocorrosion of Stellite; Nordtribe: Trondheim, Norway, 2012.

18. Lorenz, H.; Laudon, M.; Renaud, P. Mechanical characterization of a new high-aspect-ratio near UV-photoresist. Microelectron. Eng. 1998, 41-42, 371-374.

19. Chung, S.W.; Ackler, H. Material characterization of carbon-nanotube-reinforced polymer composite. Electron. Mater. Lett. 2006, 2, 175-181.

20. Marmur, A. Wetting on hydrophobic rough surfaces: To be heterogeneous or not to be. Langmuir 2003, 19, 8343-8348.

21. Marquez-Velasco, J.; Vlachopoulou, M.; Tserepi, A.; Gogolides, E. Stable superhydrophobic surfaces induced by dual-scale topography on SU-8. Microelectron. Eng. 2010, 87, 782-785.

(C) 2015 by the authors; licensee MDPI, Basel, Switzerland. This article is an open access article distributed under the terms and conditions of the Creative Commons Attribution license (http://creativecommons.org/licenses/by/4.0/). 